\title{
Research on Effectiveness of Ride-sharing Service in China Based on
}

\author{
PEST Analysis \\ Ruixue Guo ${ }^{\mathrm{a}^{*}}$, Zhili Liu ${ }^{a}$, and Jing Cai ${ }^{b}$ \\ ${ }^{a}$ Ministry of Education Key Laboratory of Complex Systems Theory and Technology of \\ Urban Traffic, Beijing Jiaotong University, China \\ ${ }^{\mathrm{b}}$ China Waterborne Transport Research Institute, China \\ * Corresponding author: Ruixue Guo, 15125740@bjtu.edu.cn
}

\begin{abstract}
Currently, sharing economy has penetrated into all fields in China, among which ride-sharing has demonstrated a leading role and has greatly changed the traffic mode. We briefly describe the definition, development courses, and the typical forms of China's ride-sharing in the beginning. Secondly, we use PEST analysis to understand the macro environment of ride-sharing in China in aspects of politics, economics, society, and technology, then numerical effectiveness was discussed based on PEST analysis. Finally several suggestions were proposed to promote informationization of ride-sharing service in China.
\end{abstract}

Keywords : sharing economy, ride-sharing service, PEST analysis, information technology, effectiveness, traffic, Internets of Vehicle

\section{Introduction}

Sharing economy is defined as a total economic activity that using the Internet and other modern information technologies to integrate and share vast amounts of decentralized idle resources, and to meet the diversified demand. ${ }^{1}$ China's sharing economy development started later than foreign countries, but it has penetrated into all fields like the knowledge sharing, life service, finance, traffic area, logistics etc. Ride-sharing service, the sharing economy in traffic area, has greatly changed tradition traffic mode in China.

\subsection{Overview of ride-sharing service}

Ride-sharing service refers to the sum of all intelligent travel modes that integrate social idle traffic resources like cars, seats, and driving skills, efficiently match traffic supply and demand using big data, and ultimately realize the sharing ability of travel based on Internet platform in the background of sharing economic. ${ }^{2}$

The development of ride-sharing service in China can be classified into three stages:

(1) The starting stage (2010.9-2012.12): Based on a combination of location-based service 
technology and mobile client, more than ten ride-sharing platforms emerged in the first-tier cities due to the serious problems of "difficulty in taking a taxi".

(2) The competition stage (2013.1-2015.2): Supported by the hot capital, both the scale and competition of ride-sharing platforms increased rapidly and further expanded into second-tier and third-tier cities. The strategic merger of Didi and Kuaidi occupied a leading position.

(3) The differentiation stage (2015.3 - Now): The competition between each platform tends to decentralise, and differentiated variety of businesses, each platform occupies a certain share of the market segment.

\subsection{Typical forms of ride-sharing service}

(1) Common ride-sharing service

Ride-sharing platform of traditional taxi, and car rental industries represented by Shouqi and Shenzhou forms B2C mode. This asset-heavy mode makes service price accordingly high due to the high cost of car purchase, maintenance, driver wages and welfare, but can ensure relatively standardized service.

Private cars join the ride-sharing platform to provide for-profit service, take "quadruple agreement" which involved platform, car leasing company, labor dispatching company and passenger to conform current regulations. The essentially private ownership determines an asset-light P2P mode. According to vehicle type and driver skill level, service can be divided into luxury and economy class, which called Zhuanche and Kuaiche respectively in Chinese.

(2) Carpooling service

Private cars also provide the non-profit carpooling service. Platforms like Didi Chuxing, Uber, Dida Pinche concentrate on this field. Take Didi Chuxing as an example "Kuaiche Pinche" has both full-time and part-time driver, platform will assign other orders with the similar route to drivers after the first order. Drivers of "Shunfengche" are the part-time ones and take appointment-driven way.

(3) Self-driving service

Traditional car rental industry also gets upgraded to provide timesharing self-driving service in both B2C and P2P mode. Fun car sharing and Shenzhou take B2C mode to provide both short-term and long-term car rental, while the former one focus on new energy automobiles. Represented by PP and Youyou, they adopt the mode of P2P and integrate idle private vehicles into a platform for renting.

"ofo" bicycle-sharing plan, as the first application of sharing economy in bicycle in schoolyard, adopts B2C mode. Students who contribute their own bicycle to "ofo" will get right to use all other "ofo"bicycles. Other students have to pay for use according to travel time and distance, parking density on WeChat. The construction of those three typical forms shown in Fig.1: 


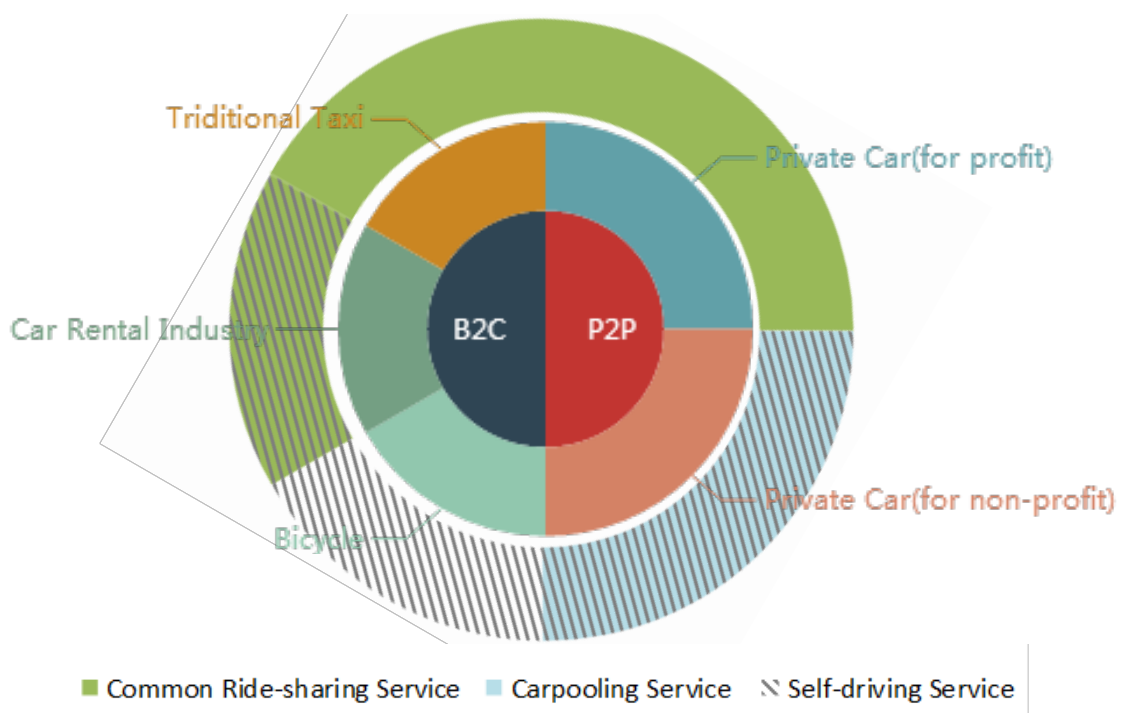

Fig. 1 -Typical forms of ride-sharing service

\section{Experimental}

\subsection{Overview of PEST analysis}

PEST analysis refers to the macro status analysis in aspects of political, economic, society and technology. It's used to well understand the current macro status of ride-sharing service in China during this chapter and numerical effectiveness will be discussed in next section.

\subsection{PEST analyses on ride-sharing}

(1) Politics factor

In October 2015, the Ministry of Transport of the People’s Republic of China announced that a new regulation will be gradually implemented in reform taxi management right. Meanwhile, a new draft regulation for "Zhuanche” defined it as car-hailing service. Carpooling also got encouraged in 2013 by Beijing Municipal Transport Commission of transport.

(2) Economic factor

In 2015, the overall market scale of sharing economy is about RMB1956 billion which including RMB1810 billion in transaction and RMB146 billion in financing. Against this backdrop, ride-sharing platforms like Didi Chuxing, Shenzhou, Uber, Yidao constantly launch financing activities.

(3) Society factor

Traditional cruising taxi service and the way of beckoning alongside of the road make a low effectiveness of demand match between drivers and passengers, denied boarding and poor service supervision become common. The ravel unmet demand cause major preferences to buy private cars, however, you have to endure a series of regulation like license-plate lottery, odd-and-even license plate rule, charge congestion and parking fee and etc.

(4) Technology factor

During the 12th five-year plan period, development of technology for a new generation of 
mobile communication and Internet, Internet of things, cloud computing, high-end software and servers got high priority. Information technology such as Internet, smart mobile terminal technology, location-based service(LBS), big data and intelligent dynamic allocation promotes the birth and development of ride-sharing service, as shown in Fig.2.

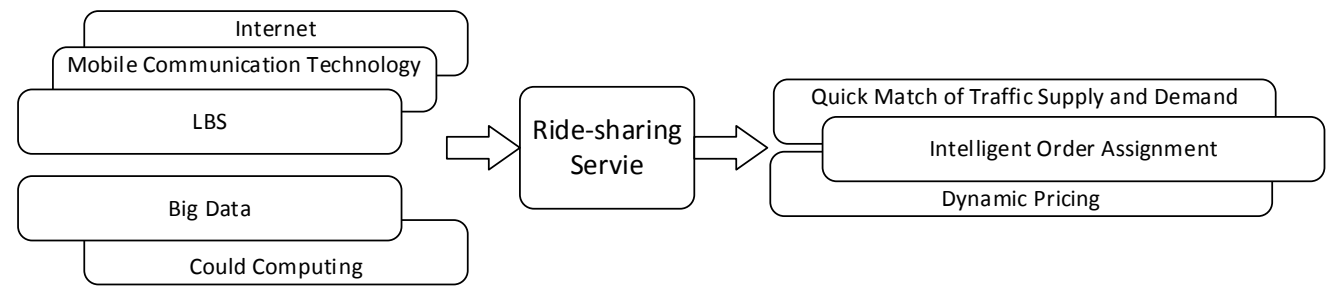

Fig. 2 -Typical technologies forms and applications of ride-sharing service

\section{Results and Discussion}

(1) Political effectiveness: ride-sharing service promotes transformation and upgrading in traditional transportation

A large number of private cars join in ride-sharing service make contributions to taxi management reform, traditional car rental companies and vehicle manufacturers also get transformed and upgraded, thus multiple formats coexist situation forms. However, the dynamic and relevant cheap pricing standard has produced a shock to taxi industry which is undergoing reform. Operating license assignment and integrated supervision become a challenge during this coexist situation.

(2) Economic effectiveness: ride-sharing service increases employment income

Fragmented time makes people pursuit more flexible employment mode, ride-sharing service right meets this demand and cultivates large scale free-lancers. The total registered drivers of Shunfengche are about 10 million, 70.3\% of the total in Didi Chuxing by the end of January 2016. According to Fig. 3, Zhuanche passengers and Taxi drivers have high consistency in willings to be a part-time driver. Another survey report shows about $40 \%$ Zhuanche drivers can get RMB1000 to RMB 3000 increases per month of income, part-time drivers have more significant income increases, as shown in Fig. 4.
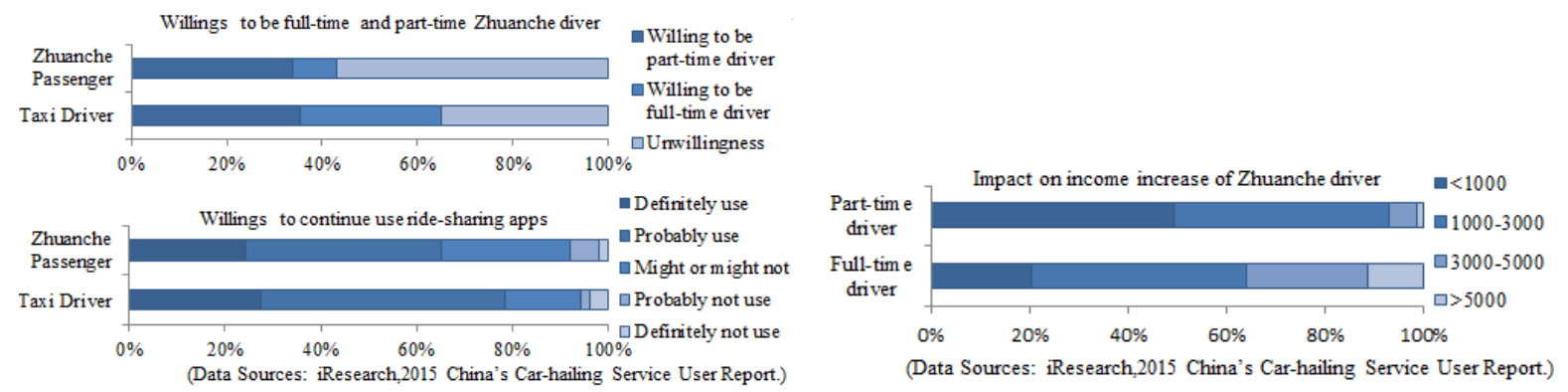

Fig. 3 - Willing towards ride-sharing service Fig. 4 - Impact on income increase of Zhuanche driver

(3) Social effectiveness: ride-sharing service changes traffic and environmental condition According to 2015 Big Data Report in China’s Smart Travel, issued by Didi Chuxing, shows 
that carpooling can reduce 1.143 million travel volumes per day in $2015,{ }^{3}$ which in other words the equivalent transport capacity can be provided. And the first time "Shunfengche" participates in the Spring Festival transport season, 1.9 million passengers were delivered home, which equivalent to run 2568 additional 8-car bullet trains. Ride-sharing service, comply with the "Supply-side Reform”, maximize transport capacity supply by integrating idle private cars rather than purchasing ones.

For the Zhuanche passengers who have private cars, the every-day travel by private cars were significantly cut by $9.2 \%$, and 5 days a week travel and noise behavior were respectively reduced by $4.6 \%$, $4.1 \%$, while 1 to 3 days a week travel were increased by $3 \%$. While for those who don't own private cars, ride-sharing service positively reduced their vehicle purchase demand by $15.24 \%$, as shown in Fig. 5. This phenomenon indicates that ride-sharing service contributes low-frequency driving and rational purchasing demand for private car.

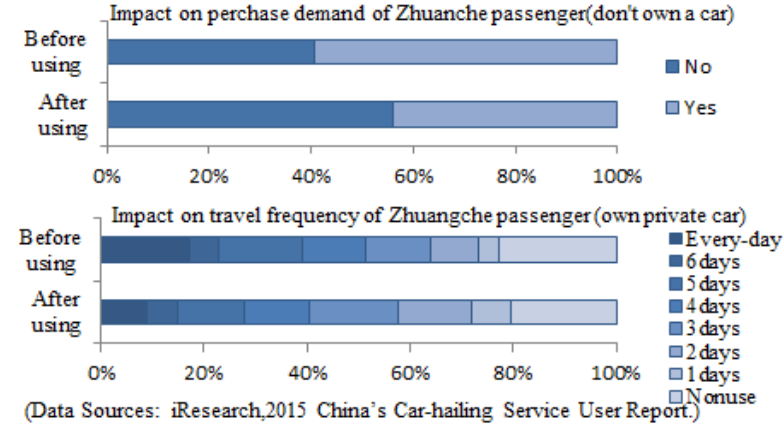

Fig. 5- Impact on travel behaviour

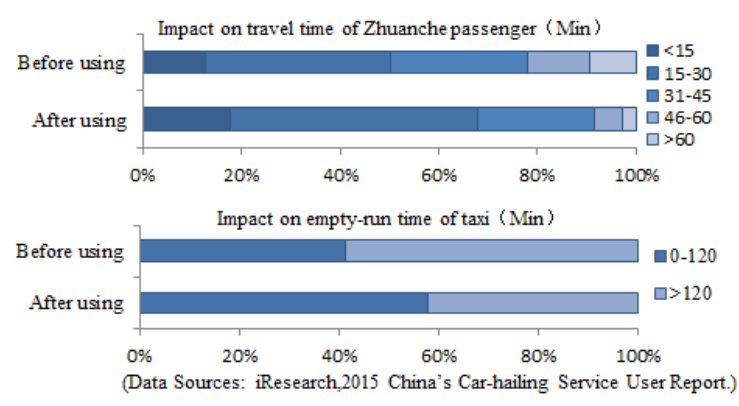

Fig. 6- Impact on travel time

According to Fig. 6, empty-run time of a taxi that longer than 2 hours, and travel time that longer than 30 minutes for the "Zhuanche" passenger were dropped by $17 \%$ or so through ride-sharing service. Thus, noneffective cruising of taxi may increase congestion of the whole traffic system, by cutting this interference, ride sharing service can meet higher travel demand for instant response and smooth driving.

Furthermore, the travel volume that carpooling reduced can save 510 million liters gasoline one year, which equivalent to reduce 13.55 million tons of carbon emissions, increases 1.13 billion ecological compensation quantities in tree planting. Highly regarded in ride-sharing service we found above will make this environmental contribution further strengthen.

(4) Technical effectiveness: ride-sharing service promotes ecological formation of Internet of Vehicles

As shown in Fig. 2, advanced technologies have widely applied in transportation service, tracing by carrying tools, ride-sharing service also boosts upstream vehicle manufacturing industry. According to iiMedia Research, China’s Internet of Vehicles markets have reached RMB110 billion in 2014, RMB 156 billion in 2015, ${ }^{4}$ analysis points out that Internet of Vehicles is still in primary stage, ecology system has not been formed yet. Low market 
appeals, low utilization of monitoring data and high costs of reinstalling after manufacture become the hinder factors. Take the "partner business plan" launched by Didi Chuxing for example, all participants can obtain a new car from automobile manufacturers that cooperated with Didi Chuxing with only pay a deposit under RMB 20000, after signing contract with labor service company, participants can receive the order. This strategy can effectively resolve above problems and establish ecological chain of Internet of Vehicles for vehicle manufacturers.

\section{Conclusion}

Based on PEST analysis, it can be seen ride-sharing service has created positive effectiveness, but more attention should be paid in following terms. Firstly, ride-sharing services for children, elder, the disabled, and pregnant woman lack enough attention. Besides, ride-sharing service mainly focused on motor vehicle, preliminary involved bicycles, further development should be emphasized on traffic structure optimization. Big data contain personal preferences and national macro trends, technology must be strengthened to ensure security.

\section{References}

1. State Information Center. Cognize sharing economy: connotation characteristics, driving force, impact, misunderstanding and development trend .J.E-Government, 4(2016):2-3.

2. State Information Center. China sharing economy development report : status quo, problems and challenges, development trend ,J. E-Government,4 (2016) :18-19.

3. http://www.imxdata.com/archives/1583(2.6.2016.)

4. http://www.iimedia.cn/41731.html(2.6.2016.) 